\title{
Experience with low-alpha lattices at the Diamond Light Source
}

\author{
I. P. S. Martin, ${ }^{1,2}$ G. Rehm, ${ }^{1}$ C. Thomas, ${ }^{1}$ and R. Bartolini ${ }^{1,2}$ \\ ${ }^{1}$ Diamond Light Source, Oxfordshire, OX11 ODE, United Kingdom \\ ${ }^{2}$ John Adams Institute, University of Oxford, OX1 3RH, United Kingdom
}

(Received 4 January 2011; published 13 April 2011)

\begin{abstract}
In this paper we present the experience at Diamond Light Source in the design, implementation, and operation of low momentum compaction factor lattices for the generation of short x-ray pulses and coherent $\mathrm{THz}$ radiation. The effects of higher-order terms in the expansion of the momentum compaction factor on beam dynamics are reviewed from a theoretical point of view, and the details of both high- and low-emittance solutions at Diamond are discussed. Measurements taken to characterize the lattices under a variety of machine conditions are presented, along with the practical limitations that exist as the momentum compaction factor is made to approach zero.
\end{abstract}

DOI: 10.1103/PhysRevSTAB.14.040705

PACS numbers: 41.60.Ap, 41.75.Fr, 29.27.Bd

\section{INTRODUCTION}

Electron storage rings can be operated in a quasiisochronous lattice in order to generate picosecond long electron bunches [1]. For a synchrotron radiation source, this is desirable for two primary reasons. First, a reduction in electron bunch length implies a corresponding reduction in x-ray pulse duration, improving the temporal resolution achievable when used for pump-probe or time-of-flight experiments. Second, a reduction in bunch length extends the wavelength range in which the electron bunch emits coherently towards the THz/far infrared region of the electromagnetic spectrum. While the majority of electron storage rings are not optimized for the production of such short electron bunches, with an appropriate tuning of the lattice optical functions it is possible to minimize the momentum compaction factor (alpha) in order to store short electron bunches with good lifetime and good injection efficiency. These so-called low-alpha lattices have been implemented at a number of light sources worldwide (see, for example, [2-12]), with several offering dedicated user time when operating in this mode $[6,7,13]$.

The low-alpha mode of operation is known to suffer from an increased sensitivity to single bunch longitudinal instabilities driven by coherent synchrotron radiation, the threshold for which decreases with alpha [14,15]. These effects typically limit the charge that can be stored in a single bunch to tens of $\mu \mathrm{A}$. For the short-pulse users, the price to pay for a reduction in pulse duration is therefore a reduction in operational bunch current of more than 2 orders of magnitude compared to standard operation.

In 2005, a program for the generation of short radiation pulses was set up at Diamond [16,17], concentrating, in

Published by American Physical Society under the terms of the Creative Commons Attribution 3.0 License. Further distribution of this work must maintain attribution to the author(s) and the published article's title, journal citation, and DOI. particular, on the low-alpha mode of operation. After a minimal hardware upgrade, the first low-alpha operation was achieved in 2008 [18], with the first user time in 2009 for pump-probe experiments [19]. In this paper we present the experience at Diamond Light Source in the design, implementation, and operation of low-alpha lattices. In Sec. II we review the relevant theory of operating with near-zero alpha, examining, in particular, the dependence of the longitudinal motion on the higher-order alpha terms and show new results for how they affect the momentum aperture. In Sec. III we present the two solutions for lowalpha operation implemented at Diamond, the second of which provides a low emittance close to that of standard machine operation. This allowed an assessment to be made of the effect of transverse emittance on the beam dynamics in low-alpha mode. In Sec. IV we present various beamdynamics measurements taken to characterize the lattices, and explain a mechanism by which the transverse beam motion grows in inverse proportion to alpha. First measurements of coherent emission at Diamond in the mm-wave region of the electromagnetic spectrum are also discussed. Conclusions and future perspectives are discussed in Sec. V.

\section{BEAM DYNAMICS WITH LOW MOMENTUM COMPACTION FACTOR}

The equilibrium bunch length of an electron beam in the "zero-current" limit scales with the square root of alpha. Modern third generation light sources operate with alphas of the order of few units in $10^{-4}$ with bunch length in the ten ps rms range or longer. A precise control of alpha is necessary to further reduce it and, correspondingly, the bunch length.

Electron storage rings with low alpha present additional challenges for the lattice optimization. A complicated beam dynamics must be controlled in the longitudinal plane, while ensuring sufficient dynamic and momentum aperture to allow electrons to be injected with good 
efficiency and stored with good lifetimes. In this section we discuss how the longitudinal motion becomes increasingly nonlinear as alpha is reduced, describe the various regimes for the longitudinal motion, and discuss how the momentum acceptance is affected by the higher-order terms in alpha.

\section{A. Momentum compaction factor}

Alpha is typically expanded as a power series in momentum deviation $(\delta=\Delta p / p)$,

$$
\alpha(\delta)=\alpha_{1}+\alpha_{2} \delta+\alpha_{3} \delta^{2}+O(\delta),
$$

where the leading order terms can be calculated using the formulas

$$
\begin{gathered}
\alpha_{1}=\frac{1}{\ell_{0}} \oint \frac{\eta_{1}(s)}{\rho} d s \\
\alpha_{2}=\frac{1}{\ell_{0}} \int \frac{\eta_{1}{ }^{\prime}(s)^{2}}{2}+\frac{\eta_{2}(s)}{\rho} d s \\
\alpha_{3}=\frac{1}{\ell_{0}} \int \eta_{1}{ }^{\prime}(s) \eta_{2}{ }^{\prime}(s)-\frac{\eta_{1}(s) \eta_{1}{ }^{\prime}(s)^{2}}{2 \rho}+\frac{\eta_{3(s)}}{\rho} d s
\end{gathered}
$$

Here, $\rho$ is the dipole bend radius, $\ell_{0}$ is the ring circumference, $\eta$ is the dispersion, $\eta^{\prime}$ is its spatial derivative, and the subscripts 1, 2, and 3 refer to the order in the expansion with momentum deviation. In order to minimize $\alpha_{1}$ the storage ring linear optics needs to be tuned such that the integrated dispersion inside the bending magnets is close to zero. As $\alpha_{1}$ is reduced it is essential to simultaneously minimize $\alpha_{2}$ so that adequate momentum aperture can be maintained [1]. This can be done using appropriately placed sextupoles. Crucially, however, once both $\alpha_{1}$ and $\alpha_{2}$ have been minimized, the next highest-order terms in the expansion for alpha quickly become dominant and their effects on the longitudinal dynamics must also be considered.

\section{B. Longitudinal beam motion}

For highly relativistic electron beams, and in the absence of radiation damping and quantum fluctuations, the equations governing the longitudinal motion can be written as

$$
\begin{gathered}
\delta^{\prime}(\varphi)=\frac{e V_{\mathrm{rf}}}{2 \pi E_{0}}\left[\sin \varphi-\sin \varphi_{s}\right] \\
\varphi^{\prime}(\delta)=h\left(\frac{\Delta \ell}{\ell_{0}}+\alpha_{1} \delta+\alpha_{2} \delta^{2}+\alpha_{3} \delta^{3}\right),
\end{gathered}
$$

where $E_{0}$ is the electron energy, $\varphi_{s}$ is the synchronous phase, $h$ is the harmonic number, $V_{\mathrm{rf}}$ is the cavity voltage, and the term $\Delta \ell / \ell_{0}$ represents all momentum-independent path lengthening effects. These equations can be derived from the Hamiltonian

$$
\begin{aligned}
H(\varphi, \delta)= & h\left[\frac{\Delta \ell}{\ell_{0}} \delta+\frac{\alpha_{1}}{2} \delta^{2}+\frac{\alpha_{2}}{3} \delta^{3}+\frac{\alpha_{3}}{4} \delta^{4}\right] \\
& +\frac{e V_{\mathrm{rf}}}{2 \pi E_{0}}\left[\cos \varphi-\cos \varphi_{s}+\left(\varphi-\varphi_{s}\right) \sin \varphi_{s}\right]
\end{aligned}
$$

The longitudinal motion has stable and unstable fixed points which occur when both $\varphi^{\prime}=0$ and $\delta^{\prime}=0$. The fixed points lie at phases $\varphi=\varphi_{s}$ and $\varphi=\pi-\varphi_{s}$, and the momentum deviations of the fixed points are given by the roots of Eq. (6), i.e., when

$$
\frac{\Delta \ell}{\ell_{0}}+\alpha_{1} \delta+\alpha_{2} \delta^{2}+\alpha_{3} \delta^{3}=0 .
$$

The momentum-independent path lengthening term can be considered to be zero at low chromaticity [20], since the contribution from closed orbit distortions to path lengthening can be offset by an adjustment of the central $\mathrm{rf}$ frequency, which in practice is always performed. Under this assumption, there exist three solutions to Eq. (8), and for all these solutions to be real the condition $\alpha_{2}^{2}>4 \alpha_{1} \alpha_{3}$ must be satisfied.

For low-alpha operation, we are interested in understanding the influence of $\alpha_{3}$ on the longitudinal motion when $\alpha_{1}$ is reduced from an initially large value towards zero or to negative values. A careful optimization of the transverse dynamics (see below) allows us to assume that $\alpha_{2}$ has been corrected to zero. After setting $\varphi_{s}=0$, we consider only the case where $\alpha_{3}<0$; the case of positive $\alpha_{3}$ is simply a reversal of these results.

Initially, for large positive $\alpha_{1}$, the longitudinal motion is described by the standard rf bucket. There are three stable regions in the longitudinal phase space, the on-momentum bucket centered at $(0,0)$ plus two off-momentum buckets centered at $\left[\pi, \pm\left(-\alpha_{1} / \alpha_{3}\right)^{1 / 2}\right]$. The separatrices for these buckets are shown in Fig. 1. As $\alpha_{1}$ is reduced, the three stable regions approach each other and the shapes of the buckets are distorted until they eventually merge. This is the transition between the conventional rf bucket regime into the alpha-bucket regime. As $\alpha_{1}$ is further reduced with respect to $\alpha_{3}$, the three buckets split again, this time with the unstable fixed points swapped such that the two buckets centered at $\left[\pi, \pm\left(-\alpha_{1} / \alpha_{3}\right)^{1 / 2}\right]$ share the one at $(\pi, 0)$, and the bucket centered at $(0,0)$ now has the two unstable fixed points at $\left[0, \pm\left(-\alpha_{1} / \alpha_{3}\right)^{1 / 2}\right]$. In this regime the longitudinal and momentum acceptance for the three buckets reduces rapidly as $\alpha_{1}$ is reduced. However, a new stable region appears which encloses the two buckets centered at $\left[\pi, \pm\left(-\alpha_{1} / \alpha_{3}\right)^{1 / 2}\right]$ for which undamped particles will follow trajectories enclosing the smaller buckets. As $\alpha_{1}$ passes through zero, the offmomentum solutions to Eq. (8) become imaginary and the bucket centered at $(0,0)$ disappears. At the same time, the two buckets centered at $\left[\pi, \pm\left(-\alpha_{1} / \alpha_{3}\right)^{1 / 2}\right]$ merge to form a single on-momentum bucket centered 

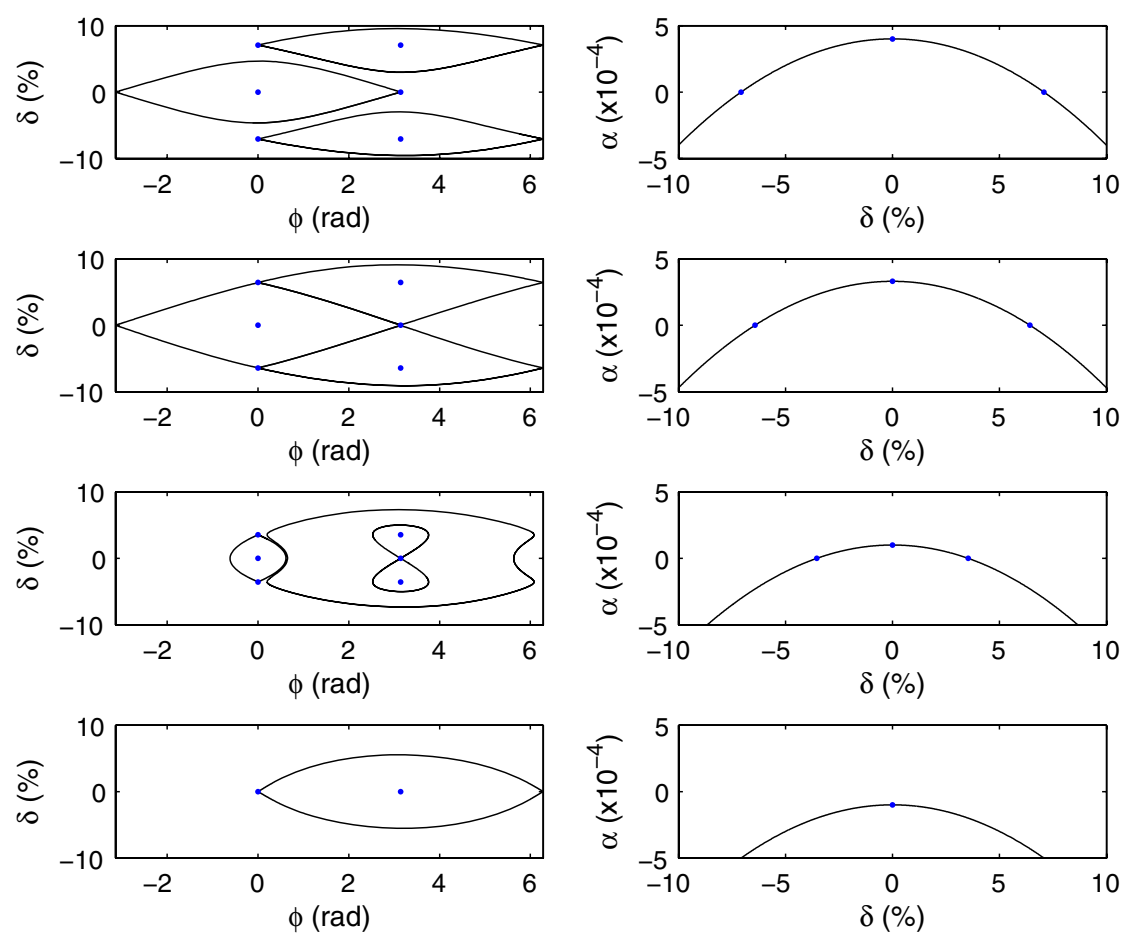

FIG. 1. Figure showing how the separatrices between stable and unstable motion vary as $\alpha_{1}$ is reduced from positive to negative values for the case where $\varphi_{s}=0, \alpha_{2}=0$, and $\alpha_{3}<0$. The right-hand plots show the corresponding values for alpha. The four stages illustrated are rf-bucket regime (top), transition (middle top), alpha-bucket regime (middle bottom), and negative alpha (bottom).

at $(\pi, 0)$; alpha is now entirely negative and the stable rf phase has switched from $\varphi=0$ to $\varphi=\pi$.

It is interesting to note that, had we chosen to operate with positive $\alpha_{3}$, the situation described above is reversed, i.e., as $\alpha_{1}$ is made to pass from positive to negative values it is the bucket centered at $(0,0)$ that splits into two, rather than the two off-momentum buckets that merge to form a single on-momentum bucket. This transition can occur smoothly without loss of beam [13].

Transition between the $\mathrm{rf}$ and alpha-bucket regimes occurs when the Hamiltonians for the separatrices of the three buckets are equal, namely, when

$$
H(\pi, 0)=H\left(0, \pm \sqrt{-\alpha_{1, \text { transition }} / \alpha_{3}}\right)
$$

which upon substitution into Eq. (7) eventually reduces to

$$
\alpha_{1, \text { transition }}=-2 \alpha_{3} \varepsilon_{\mathrm{rf}}^{2},
$$

where $\varepsilon_{\mathrm{rf}}$ is the energy acceptance given by the classical result for the first order solution of Eq. (9) [21]. This transition point can be used as a criterion for good longitudinal and momentum aperture, i.e., to stay in the rf-

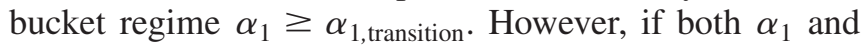
$\alpha_{3}$ have the same sign then the off-momentum fixed points are imaginary and this condition no longer applies. In this case the $\alpha_{3}$ term creates a perturbation on the maximum stable momentum oscillation of the linear rf bucket. The momentum acceptance can be found by equating the value of the Hamiltonian at the unstable fixed point to the value at the phase $\varphi=\varphi_{s}$, i.e.,

$$
H(0,0)=H\left(\pi, \delta_{\max }\right)
$$

which leads to the result

$$
\delta_{\max }^{2}+\frac{\alpha_{3}}{2 \alpha_{1}} \delta_{\max }^{4}=\varepsilon_{\mathrm{rf}}^{2} .
$$

In this regime, the $\alpha_{3}$ term serves to increase the momentum aperture above the one found from the classical first order solution, and particles exhibit regular motion out to large momentum and phase deviations.

\section{Transverse dynamics}

Optimization of the transverse dynamics in low-alpha lattices poses additional challenges with respect to the usual optimization. Good dynamic and momentum apertures still have to be provided, while simultaneously setting $\alpha_{2}$ to zero and ensuring that higher-order terms remain within limits that do not spoil the lattice performance. The task of optimizing the sextupole strengths is made more demanding by the reduced freedom to control the linear dispersion function. As an example, a reduction in $\alpha_{1}$ can lead to negative dispersion appearing at the sextupole locations, which in turn makes it necessary to implement a switch in polarity of these magnets for them to remain effective. 


\section{LOW-ALPHA LATTICES AT DIAMOND}

Low-alpha lattices for Diamond were developed targeting several objectives simultaneously. The main goals were to have small, variable $\alpha_{1}$, sufficient on-momentum dynamic aperture to be able to inject directly into the lattice without shifting the working point and changing the optics, sufficient off-momentum dynamic aperture for good lifetime and injection, small beta function in the insertion device (ID) straight sections, large horizontal beta function at the injection point and small, controllable chromaticity (both transverse and longitudinal). Additionally, it was desirable to have low emittance in order to maximize the source brightness (relevant for the short-pulse x-ray users).

The Diamond storage ring is a double-bend achromat lattice, for which the standard approach to achieve low alpha is to introduce negative dispersion in the straight sections [2,3,6-10]. For this solution the dispersion is made to cross from positive values between the bending magnets to negative values in the straights, thus minimizing Eq. (2) at the expense of a significant increase in horizontal emittance. However, an alternative solution is to keep the dispersion positive everywhere except in the center of the bending magnets $[17,22,23]$. This allows both $\alpha_{1}$ and the emittance to be minimized simultaneously; both these low-alpha solutions have been implemented at Diamond $[18,24]$. A summary of the main storage ring parameters in each case is given in Table I, and the optical functions for one super-period for the two lattices are given in Figs. 2 and 3.

There are several key differences between the two solutions. First, the horizontal integer tune point for the lowemittance lattice is much larger than for the high-emittance lattice at 29 compared to 21 (the standard user optics has an integer tune point of 27). This increase in horizontal tune leads to an increase of the natural chromaticity, which combined with the reduction in dispersion at the sextupole locations results in a marked increase in the required sextupole strengths $(S)$. This problem is exacerbated by the need to minimize the longitudinal chromaticity $\alpha_{2}$, a term which is proportional to $\eta_{1}{ }^{3} S$ and is predominantly controlled by

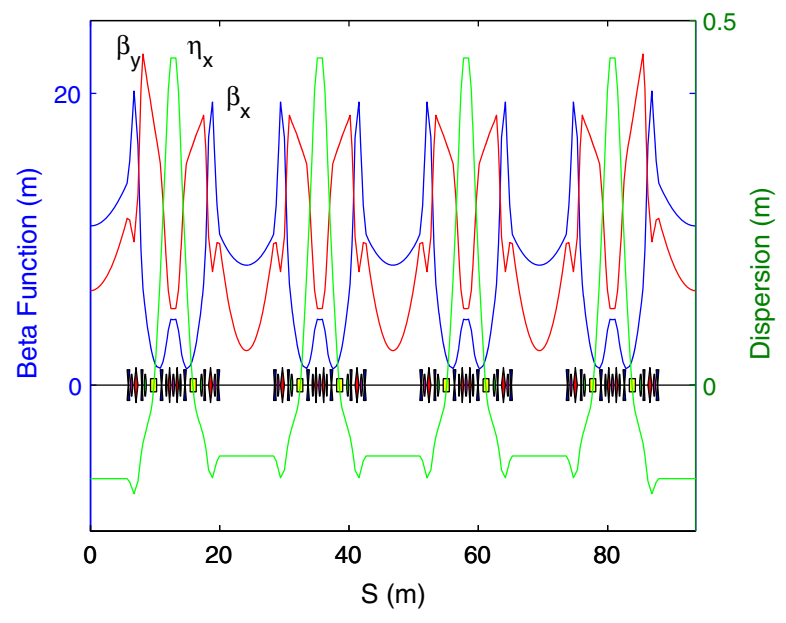

FIG. 2. Optical functions for the high-emittance lattice.

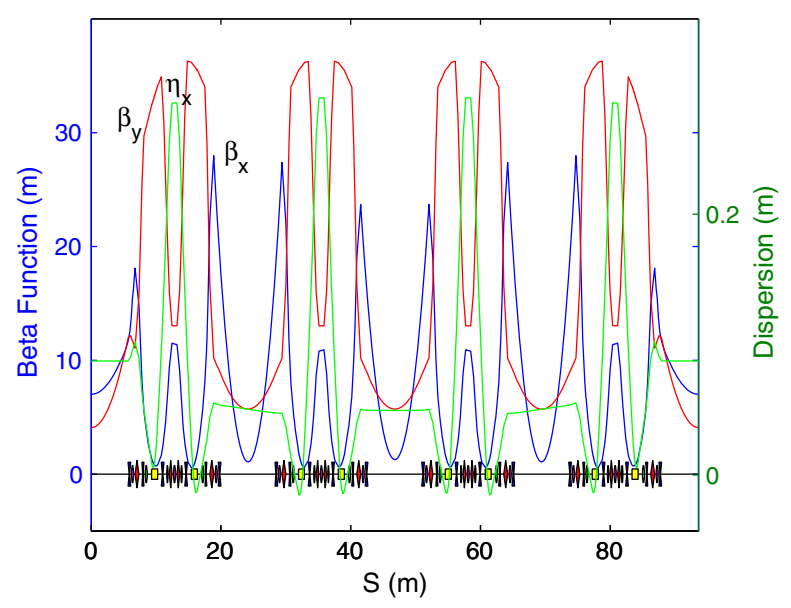

FIG. 3. Optical functions for the low-emittance lattice.

the focusing sextupoles in the center of the arcs, where the peak dispersion is considerably reduced in the low-emittance lattice. Peak sextupole strengths for the low-emittance lattice are therefore unavoidably stronger than those required for the high-emittance lattice $\left(31.3 \mathrm{~m}^{-3}\right.$ compared to $23.6 \mathrm{~m}^{-3}$, respectively),

TABLE I. Main design parameters of the two low-alpha lattices.

\begin{tabular}{lcc}
\hline \hline $\begin{array}{l}\text { Parameter } \\
\text { emittance }\end{array}$ & $\begin{array}{c}\text { High emittance lattice } \\
35.2 \mathrm{~nm} \mathrm{rad}\end{array}$ & $\begin{array}{c}\text { Low-emittance lattice } \\
4.4 \mathrm{~nm} \mathrm{rad}\end{array}$ \\
\hline$\alpha_{1}$ (with/without sextupoles) & $-3 \times 10^{-6}$ & $-1 \times 10^{-5}$ \\
$\alpha_{2}$ (with/without sextupoles) & $-6 \times 10^{-5} / 0.0116$ & $-2 \times 10^{-5} / 0.005$ \\
$\alpha_{3} / Q_{y}$ & $-0.0426 /-0.193$ & $0.004 / 0.008$ \\
$Q_{x}$ & $21.150 / 12.397$ & $29.390 / 8.284$ \\
Natural $\xi_{x} / \xi_{y}$ & $-37 /-26$ & $-66 /-43$ \\
$\beta_{x} / \beta_{y}$ ID & $8.2 \mathrm{~m} / 2.4 \mathrm{~m}$ & $1.1 \mathrm{~m} / 5.7 \mathrm{~m}$ \\
Maximum $\eta_{x}$ & $0.45 \mathrm{~m}$ & $0.28 \mathrm{~m}$ \\
Natural bunch length $(3 \mathrm{MV})$ & $1.3 \mathrm{ps}$ & $2.4 \mathrm{ps}$ \\
Synchrotron frequency $(3 \mathrm{MV})$ & $346 \mathrm{~Hz}$ & $629 \mathrm{~Hz}$ \\
\hline \hline
\end{tabular}


increasing the nonlinearity of longitudinal and transverse beam motion and thus shrinking the region of stable motion. In spite of this, injection efficiencies above $40 \%$ and lifetimes above $10 \mathrm{~h}$ are regularly maintained for the user conditions.

Based on experience gained during machine development trials for the high-emittance lattice, a larger absolute value for $\alpha_{1}$ was targeted for the low-emittance solution from the beginning. There are two main reasons for this choice. First, while the larger $\alpha_{1}$ gives a longer natural (zero-current) bunch length, the bunch length at moderate bunch currents rapidly becomes independent of $\alpha_{1}$, i.e., there is no benefit in further reducing $\alpha_{1}$ once a minimum bunch length has been achieved at any given current. Second, the horizontal closed orbit stability of the electron beam is found to be inversely proportional to $\alpha_{1}$. This reduced closed orbit stability becomes important to the users when the emittance of the beam is reduced. Selecting a slightly larger $\alpha_{1}$ helps to reduce the impact of this increased sensitivity to transverse motion, without increasing the bunch length at the given operating current. These features are discussed in the following section.

The final difference to note is that the sign of $\alpha_{3}$ is positive for the low emittance and negative for the highemittance lattice. The results from Sec. II would suggest it is better to operate the low-emittance lattice at positive $\alpha_{1}$ in order to benefit from the removal of the off-momentum fixed points of motion and, hence, improved momentum aperture; however, when switching to positive $\alpha_{1}$ the sign of $\alpha_{3}$ is also found to reverse. As such, operation with negative $\alpha_{1}$ is still preferred in order to benefit from the reduced bunch lengthening with current compared to operating with positive $\alpha_{1}$.

Extensive nonlinear beam-dynamics studies were carried out on both lattices during the design stage in order to identify which solutions were expected to perform optimally and also to establish the expected performance criteria. This work assessed the dynamic and momentum apertures available to the beam, investigated the injection process and mapped out the longitudinal phase space with a full 6D tracking, complete with rf cavity and radiation damping (where appropriate). Frequency maps were also used to evaluate the quality of the lattice optimization [25]. For the studies, a combination of TRACY-II [26], ELEGANT [27], and Accelerator Toolbox [28] were used. The final solutions were found to be sufficiently robust to operate without any need for optics switches to ease injection, even after including realistic magnet field errors and physical apertures in the simulations. Plots of the available on- and off-momentum dynamic apertures, frequency map, and momentum aperture for the low-emittance lattice are reported in Fig. 4, along with alpha and the longitudinal phase space. The longitudinal dynamics for the lowemittance lattice shows strong nonlinearities and requires many terms in the polynomial expansion for alpha for an accurate description to be made. Alpha crosses from negative to positive values at $\delta=+4 \%$, meaning that Eq. (8) has two solutions and two stable regions appear
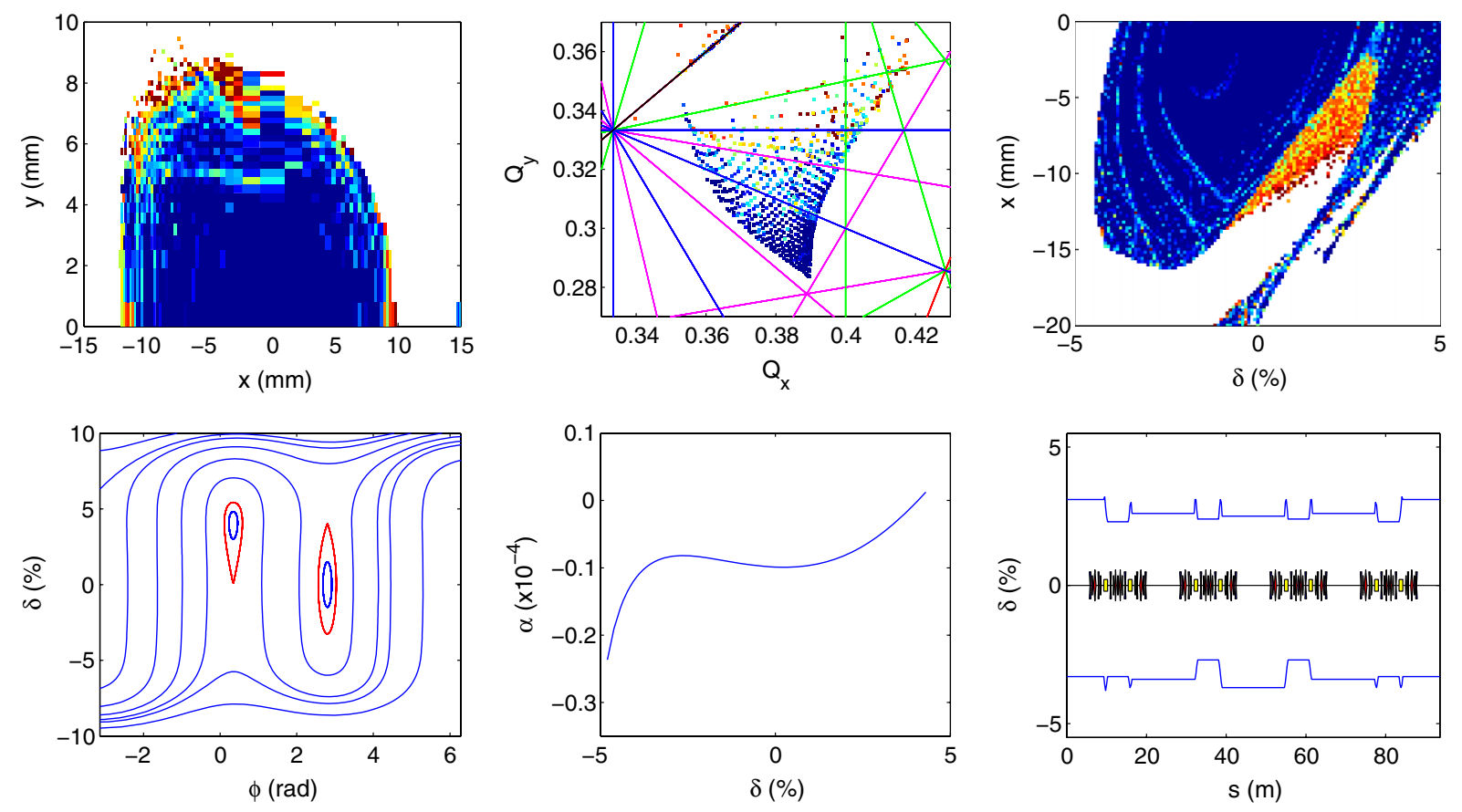

FIG. 4. On-momentum dynamic aperture (top left), frequency map (top middle), and off-momentum dynamic aperture (top right) for the low-emittance lattice. The longitudinal phase space (bottom left), momentum compaction factor (bottom middle), and momentum acceptance (bottom right) for one super period of the ring are also shown. The separatrices between stable and unstable motion are highlighted in red. 
(one on-momentum bucket centered at $\varphi=\pi-\varphi_{s}$ and one off-momentum centered at $\varphi=\varphi_{s}$ ). As a result, the low-emittance lattice operates in the alpha-bucket regime. In order to accommodate six sigma of the injected bunch from the booster $\left(\sigma_{l, \text { booster }}=26 \mathrm{~mm}\right)$, a phase acceptance of $\pm 0.82 \mathrm{rad}$ is required out to $-11.2 \mathrm{~mm}$ horizontal displacement. After $\frac{1}{4}$ of a synchrotron period, this translates into a required energy acceptance of above $\pm 4 \%$. As clear from the on- and off-momentum dynamic aperture plots in Fig. 4, it has not been possible to meet this challenging requirement. However, injection efficiencies in the range $30 \%-50 \%$ were predicted by the simulations, in remarkable agreement with the measured values for the low-emittance lattice.

\section{MACHINE MEASUREMENTS}

\section{A. Bunch length measurements}

The numerical optimization of the low-alpha lattices allowed promising solutions to be found with $\left|\alpha_{1}\right|=$ $1 \times 10^{-6}$. At Diamond this corresponds to a predicted bunch length of $1 \mathrm{ps}$ rms. Electron bunch length measurements are taken using an Optronis dual-sweep streak camera [29]. The combined static-dynamic point spread function (PSF) for this camera is estimated to be in the range 1.5-3 ps depending upon the precise details of the setup, and is a parameter which must be checked whenever bunch length measurements are to be taken. The magnitude of PSF makes bunch length measurements in low-alpha mode extremely challenging at the shorter end of the scale, where the predicted bunch lengths are less than the PSF of the camera and are therefore subject to significant systematic errors. The performance of the camera remains under active study [30].

The possibility to operate the Diamond storage ring in high- and low-emittance low-alpha modes allows the bunch lengthening with current to be directly compared for the two cases. For this measurement, $\alpha_{1}$ was held fixed at $-6 \times 10^{-6}$ and the cavity voltage was varied between 1.5 and 2.2 MV. The results are shown in Fig. 5. There is good agreement between the data sets up to a bunch current of $42 \mu \mathrm{A}$; however, for the data taken at $62 \mu \mathrm{A}$ the measured bunch length for the low-emittance lattice was longer and was observed to emit "bursting" radiation due to microbunching [31]. This earlier onset of bursting radiation for the low-emittance lattice can be explained as a direct consequence of the increase in electron density.

As expected from the theory for negative alpha [32], an initial reduction in bunch length with increasing current due to potential well distortion is observed before collective effects cause the bunch length to begin to grow again. In contrast to this, for positive alpha the bunch length grows immediately from the same zero-current (natural) bunch length. For negative alpha, the bunch lengthening onset occurs at bunch currents of only a few $\mu \mathrm{A}$, and varies with the particular values of cavity voltage and alpha. It is

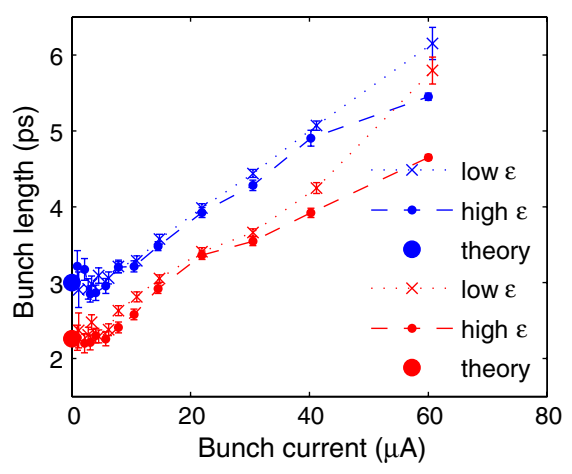

FIG. 5. Comparison between bunch length measurements for the high- and low-emittance lattices at $\alpha_{1}=-6 \times 10^{-6}$. The cavity voltage was varied between 1.5 MV (blue) and 2.2 MV (red). The theoretical natural (zero-current) bunch lengths given by Eq. (13) are marked as dots.

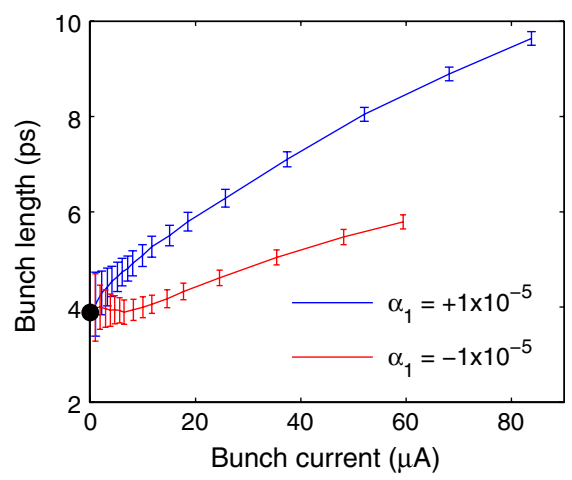

FIG. 6. Comparison between bunch length measurements made for the low-emittance lattice at $\left|\alpha_{1}\right|=1 \times 10^{-5}$. The cavity voltage was set to $1.5 \mathrm{MV}$. The theoretical natural (zero-current) bunch length given by Eq. (13) is also given.

anticipated that, for negative alpha, the energy spread will increase more rapidly with current than is the case for positive alpha; however, we have been unable to confirm this as the stored current is below the minimum value required by the $\mathrm{x}$-ray pinhole camera system for accurate measurements to be made. Figure 6 shows a comparison between bunch length measurements taken for both positive and negative alpha for the low-emittance lattice at a fixed value of $\left|\alpha_{1}\right|=1 \times 10^{-5}$ and a cavity voltage of $1.5 \mathrm{MV}$.

The raw data and fitted curves are shown for a range of machine conditions in Fig. 7. From this it is clear that the reduction in bunch length with $\alpha_{1}$ at a given rf cavity voltage is only apparent for bunch currents below $\sim 20-30 \mu \mathrm{A}$. An interesting feature from this plot is that the bunch length curves for different values of $\alpha_{1}$ only converge to a common value at a fixed cavity voltage; i.e., there is no universal relation linking bunch length to bunch current, but rather the limiting values depend on cavity voltage. Note that for the data shown in Fig. 7 the PSF was taken to be $1.7 \mathrm{ps}$ in order to give good agreement with the 


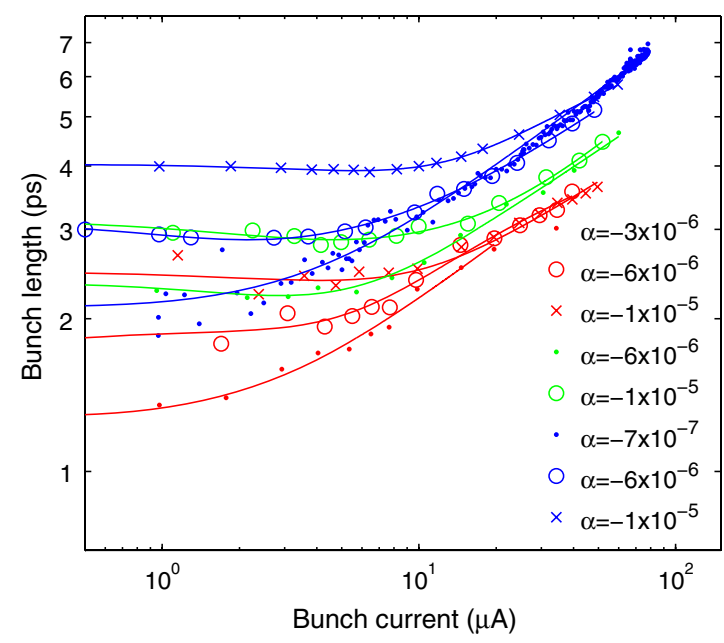

FIG. 7. Bunch length as a function of current under a variety of conditions. Data in red was measured at $3.4 \mathrm{MV}$, data in green was measured at $2.2 \mathrm{MV}$, and data in blue was measured at $1.5 \mathrm{MV}$, all using the low-emittance lattice.

zero-current bunch length extracted from the measured synchrotron frequency. This PSF is marginally above the measured value, with the discrepancy believed to be due to phase noise in either the streak camera of rf systems. A more precise theoretical understanding of the bunch length dependence on bunch current is the subject of future work, for which a multiparticle tracking code is to be extended to include the effects of coherent synchrotron radiation [33].

\section{B. Synchrotron frequency measurements}

As an alternative to streak camera measurements, the natural electron bunch length can be inferred from the synchrotron frequency:

$$
\sigma_{L}=\frac{c E_{0} \sigma_{E}}{h \cos \varphi_{s} e V_{\mathrm{rf}} f_{\mathrm{rev}}^{2}} f_{s},
$$

where $\sigma_{E}$ is the energy spread and $f_{\text {rev }}$ is the revolution frequency. This method requires some assumptions to be made about the operating machine conditions (such as beam energy, rf cavity parameters, electron energy spread); however, for the shortest electron bunches stored in the Diamond storage ring and for low bunch currents, this method is judged to be the most accurate form of bunch length measurement presently available.

To assist in the measurement of the synchrotron frequency, a phase modulator was installed for the rf system which allows synchrotron oscillations to be excited in the beam. These oscillations can then be observed at a beam position monitor (BPM) at a location of high transverse dispersion. An example of how the rf phase modulator improves the synchrotron frequency measurements is given in Fig. 8, and a measurement taken at a synchrotron frequency of $185 \mathrm{~Hz}$ is given in Fig. 9. The rf cavity voltage in this case was $3.4 \mathrm{MV}$, corresponding to natural electron

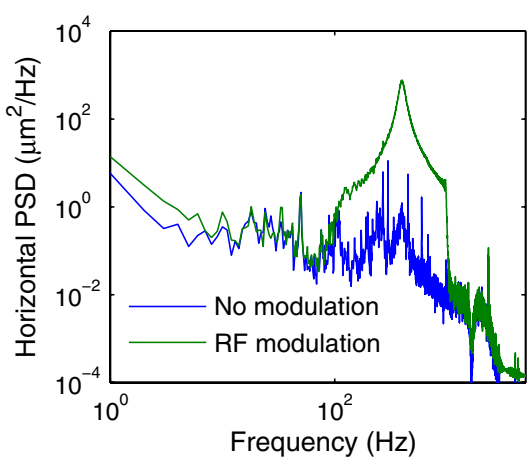

FIG. 8. Comparison of synchrotron frequency measurements made with and without rf cavity phase modulation. Beam motion was recorded at a BPM with $\eta_{x}=0.28 \mathrm{~m}$.

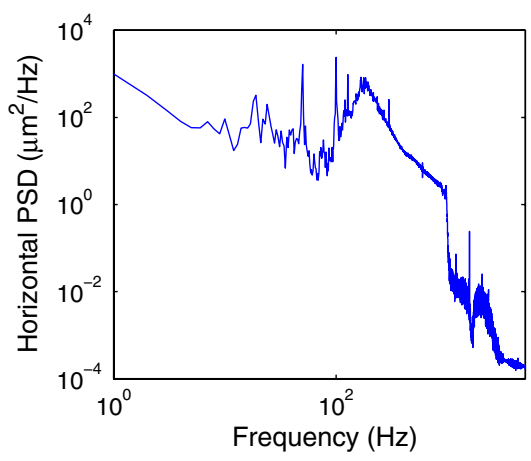

FIG. 9. Synchrotron frequency measurements made for $V_{\mathrm{rf}}=$ 3.4 MV and $\alpha_{1}=-7 \times 10^{-7}$. The corresponding natural electron bunch length is $0.6 \mathrm{ps}$.

bunch length of $0.6 \mathrm{ps}\left(\alpha_{1}=-7 \times 10^{-7}\right)$. Both these data sets were recorded using the low-emittance lattice.

\section{Coherent emission}

A further confirmation of the short bunch lengths achieved with the low-alpha lattices can be found by observing the emission at wavelengths comparable to or longer than the bunch length, at which point the bunch is expected to emit coherently. The total power radiated by the electron bunch at a given wavelength can be derived from the power emitted by a single electron $\left[P_{e}(\lambda)\right]$, the number of electrons in the bunch $\left(n_{e}\right)$, and a form factor $\left(f_{\lambda}\right)[34]$ :

$$
P(\lambda)=P_{e}(\lambda)\left[n_{e}+n_{e}\left(n_{e}-1\right) f_{\lambda}\right] .
$$

The form factor $f_{\lambda}$ is given by the square of the Fourier transform of the charge distribution and varies between 0 for an infinitely long bunch (incoherent emission) and 1 for a point source (coherent emission). For large $n_{e}$ (as is the case in electron storage rings), a clear indication that the emitted radiation is coherent is given if $P(\lambda)$ is found to be proportional to $n_{e}^{2}$. 


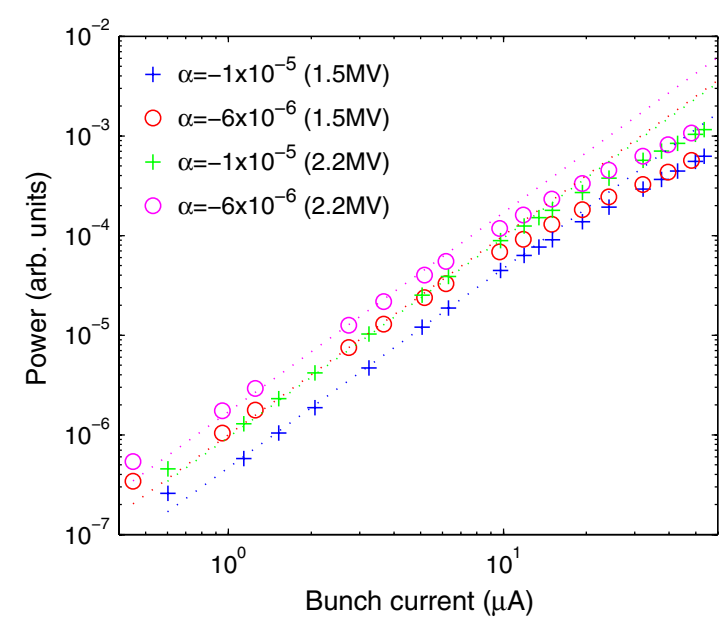

FIG. 10. Power emitted in the $60-90 \mathrm{GHz}$ bandwidth as a function of current for two values of $\alpha_{1}$. Data was measured at 1.5 and $2.2 \mathrm{MV}$.

To detect coherent emission the Diamond storage ring is equipped with a Schottky barrier diode sensitive to the 60-90 GHz bandwidth [35]. This detector has a fast response time of $250 \mathrm{ps}$ which allows the structure and evolution of the coherent emission to be studied on a turn-by-turn basis, albeit restricted to measuring $\mathrm{mm}$ wave radiation longer than the expected bunch length.

The emitted radiation in this bandwidth is shown in Fig. 10 for a variety of machine conditions for the lowemittance lattice. The radiation was emitted in a steady state, i.e., the bunch current was kept below the bursting instability threshold (found to be in the range 50-55 $\mu \mathrm{A}$ for these measurements). The data shows that the power emitted in this bandwidth at a given rf voltage is initially higher for a reduced value of $\alpha_{1}$ (when the bunch length is shorter), but as the current is increased and the bunch length becomes independent of $\alpha_{1}$ the emitted power also becomes independent of $\alpha_{1}$. As the rf voltage is increased, the bunch length is shortened and again the emitted power increases consistent with the results of Fig. 7.

Further analysis of this data shows that the differences in emitted power can be explained purely by the form factor $f_{\lambda}$ for each data point. The form factors are extracted from streak camera images recorded at the same time as the mmwave data, and show both a bunch lengthening and bunch distortion as the bunch current is increased. If the measured power is normalized to the numerically calculated form factor for $\lambda=5 \mathrm{~mm}$, then the power emitted becomes independent of machine parameters and proportional to $I_{b}^{2}$, as predicted by Eq. (14) for the case of $f_{\lambda} n_{e} \gg 1$. A selection of bunch profiles for increasing bunch current are shown in Fig. 11, and the power normalized to $f_{\lambda=5 \mathrm{~mm}}$ is shown in Fig. 12. Normalizing the mm-wave data to a form factor calculated using a Gaussian bunch of equal FWHM significantly underestimates the power emitted at higher

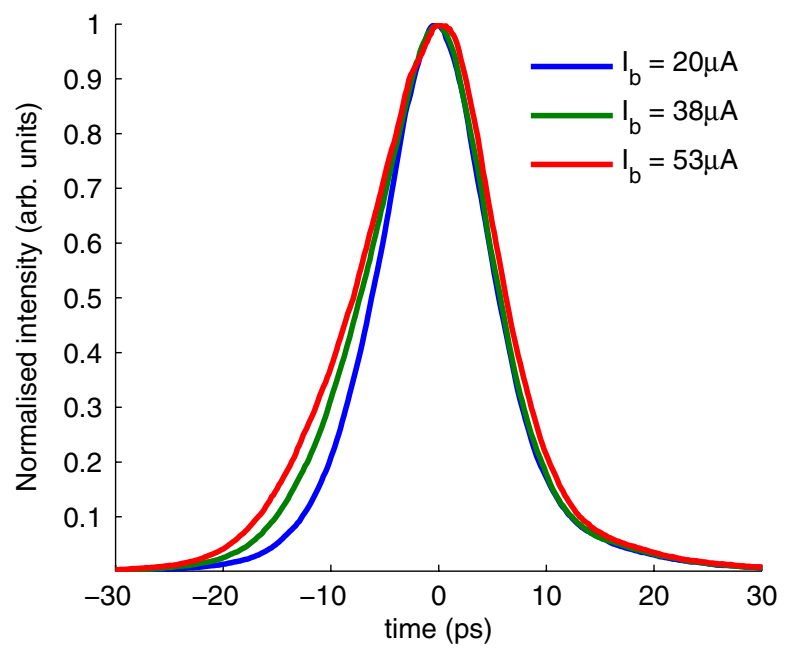

FIG. 11. Bunch profiles recorded with the streak camera for $\alpha_{1}=-1 \times 10^{-5}$ and $V_{\mathrm{rf}}=1.5 \mathrm{MV}$.

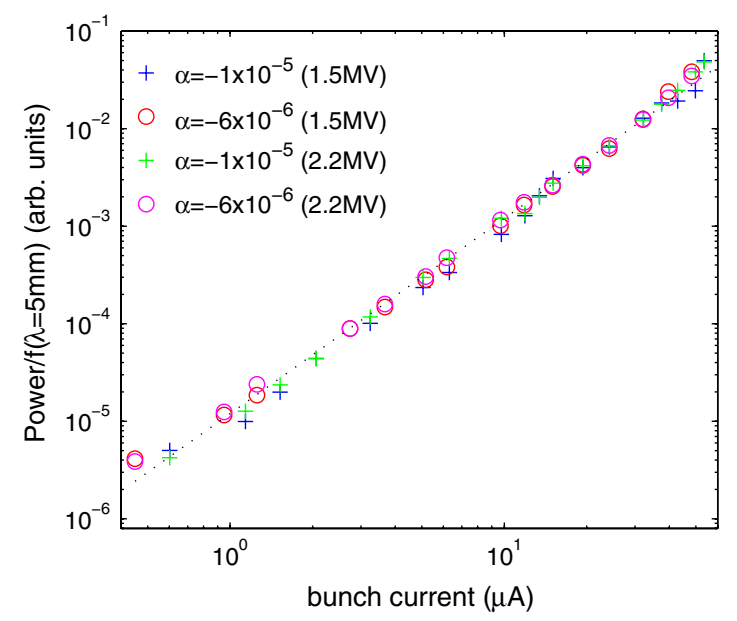

FIG. 12. Power measured with the Schottky barrier diode normalized to the form factor calculated using the bunch profile measured using a streak camera. The power normalized in this way becomes independent of machine parameters and proportional to $I_{b}^{2}$, consistent with steady-state coherent emission.

bunch currents; it is necessary to consider both a bunch lengthening and a bunch shape distortion to explain the measured data, suggesting (14) should be written as

$$
P(\lambda)=P_{e}(\lambda)\left[n_{e}+n_{e}\left(n_{e}-1\right) f\left(\lambda, n_{e}\right)\right] .
$$

\section{Orbit stability}

Increased transverse beam motion is a known problem for low-alpha lattices [8,36]. At Diamond, horizontal electron beam motion for both high- and low-emittance lattices has been measured to be greater than that of the nominal storage ring by up to 2 orders of magnitude. The beam motion is always dominated by a dispersive pattern, 


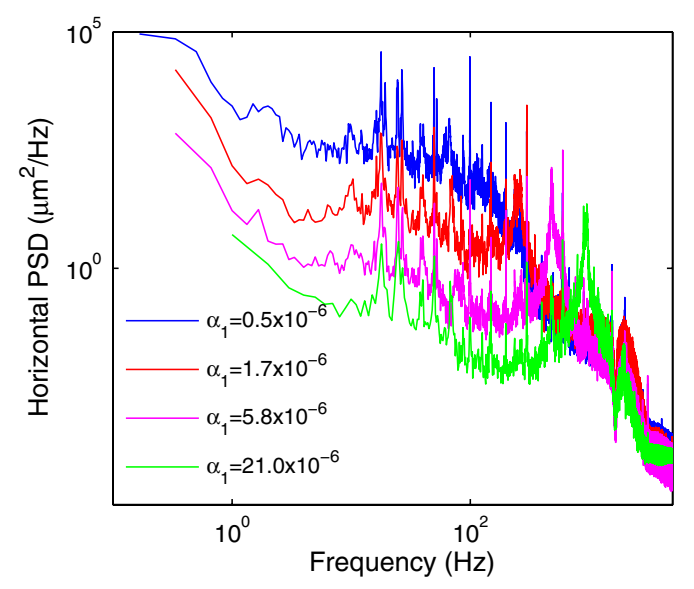

FIG. 13. Displacement PSD at a high dispersion BPM for several values of alpha.

implying the root cause of the increase in horizontal motion is related to an increased sensitivity to fluctuations in the beam energy. The vertical motion is also affected as $\alpha_{1}$ is reduced, but is less apparent due to the smaller dispersion in the vertical plane.

Shown in Fig. 13 are measurements of the horizontal electron beam displacement power spectral density (PSD) for four different values of $\alpha_{1}$. The profile of the vibrations remains constant in each case, with only the amplitude of motion and the location of the synchrotron frequency peak changing as alpha is altered. The PSD profiles closely match the measured ground motion spectrum, suggesting that the primary mechanism driving the horizontal electron beam motion is small changes to the ring circumference caused by ground vibrations. This can be understood from the definition of alpha:

$$
\alpha=\frac{\Delta \ell / \ell_{0}}{\delta} .
$$

Since the average circumference is fixed by the rf frequency, small changes to the electron path length will translate directly into changes in electron energy in inverse proportion to the momentum compaction factor. An estimate for the magnitude of path length fluctuations caused by ground motion can be made by considering quadrupole vibrations in regions of nonzero dispersion. In this case, the change in path length is given by

$$
\Delta \ell=\sum_{n} K_{n} L_{n} \eta_{n}\left\langle\sigma_{\text {quad }}\right\rangle
$$

where $K_{n}$ is the quadrupole strength, $L_{n}$ is the magnet length, $\eta_{n}$ is the dispersion at the quadrupole, and $\left\langle\sigma_{\text {quad }}\right\rangle$ is the average transverse vibration amplitude for the magnets. For Diamond, the average amplitude of quadrupole vibrations is $\sim 40 \mathrm{~nm}$, which in turn gives average path length fluctuations of $\sim 0.24 \mu \mathrm{m}$. For the case of $\alpha_{1}=-1 \times 10^{-5}$, the resulting energy fluctuations are $\delta \sim 0.04 \%$, and at a location where the dispersion is

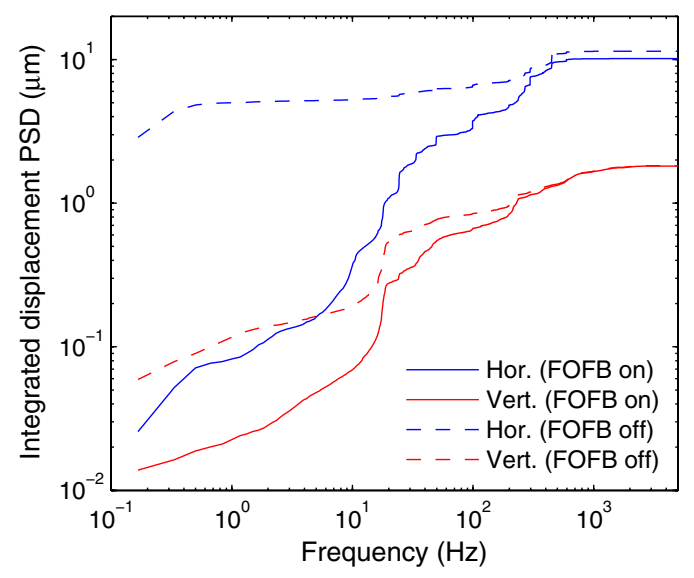

FIG. 14. Integrated beam motion with and without orbit feedback for $\alpha_{1}=-1 \times 10^{-5}$ and a cavity voltage of 1.6 MV.

$0.3 \mathrm{~m}$ this would result in $\sim 13 \mu \mathrm{m}$ horizontal beam motion, consistent with the measured integrated value, as shown in Fig. 14.

In order to suppress transverse beam motion, the fast orbit feedback (FOFB) has been set up to run with a reduced bandwidth. The integrated beam motion with and without feedback running is shown in Fig. 14. While horizontal beam motion in the range $1-100 \mathrm{~Hz}$ is above that of the nominal lattice at $3.7 \mu \mathrm{m}$, this is still well below the target $10 \%$ of beam size.

\section{E. Pinhole camera images}

Two x-ray pinhole cameras are available at Diamond for imaging the electron beam, and from these images the emittance and energy spread of the electron beam can be extracted [37]. Such measurements are subject to increased uncertainty for the low-alpha mode of operation due to the low beam currents involved and increased transverse beam motion on time scales shorter than the typical camera integration times. Optimization of the emittance measurement procedure in low alpha is ongoing; however, preliminary values of $37.2 \pm 1.6 \mathrm{~nm} \mathrm{rad}$ and $3.7 \pm 0.3 \mathrm{~nm} \mathrm{rad}$ have been recorded for the high- and low-emittance lattices, respectively.

The pinhole cameras have also been used to confirm the existence of two stable regions in the longitudinal phase space for the low-emittance lattice. For this measurement, electrons were injected into the storage ring at $\varphi=\pi-\varphi_{s}$, before slowly shifting the $\mathrm{rf}$ cavity phase to $\varphi=\varphi_{s}$ and reinjecting without losing the original stored beam. In order to separate the two beams on the pinhole camera screens, the vertical dispersion was artificially enhanced by increasing skew-quadrupole strengths. Pinhole camera images shown in Fig. 15 clearly show the existence of two stored beams separated in space due to the different beam energies and finite vertical dispersion at the pinhole camera source points. 


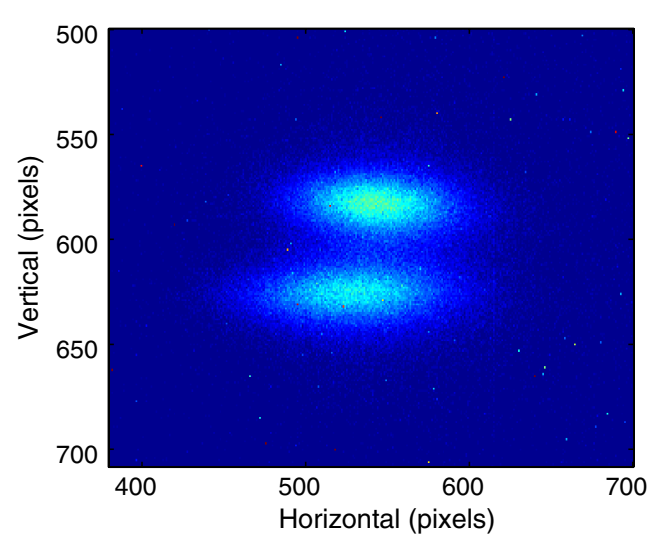

FIG. 15. X-ray pinhole camera image for the low-emittance lattices showing two stored electron beams at phases $\varphi=\pi-\varphi_{s}$ and $\varphi=\varphi_{s}$.

\section{F. Operational aspects}

Since April 2009, three dedicated four-day periods of machine time have been devoted to users of short-pulse $\mathrm{x}$ rays for pump-probe experiments. For the first user period the high-emittance lattice was applied, during which time difficulties were identified regarding the overlap of the large horizontal $\mathrm{x}$-ray beam size to the smaller pump laser beam size. Since then, the low-emittance lattice has been routinely used.

During user beam time, the machine is operated with measured alpha values of $\alpha_{1}=-1 \times 10^{-5}$ and $\alpha_{2}=1.5 \times 10^{-5}$, horizontal/vertical chromaticities of $+0.6 /-0.8$, and an emittance coupling of $0.25 \%$. A hybrid fill pattern is used, consisting of a continuous train of $8 \mathrm{~mA}$ in 686 bunches, with a single hybrid bunch of $37.5 \mu \mathrm{A}$ in the gap. In this way, the light from the hybrid bunch can be used for pump-probe experiments and sufficient charge is stored for reliable operation of the storage ring BPMs, the fast orbit feedback and the rf frequency feedback systems. Under these conditions, the hybrid bunch lifetime typically ranges from 10 to $20 \mathrm{~h}$ over the course of a single fill, during which time the hybrid bunch decays from $\sim 37.5 \mu \mathrm{A}$ to $\sim 10 \mu \mathrm{A}$.

\section{CONCLUSIONS}

The implementation of a low-emittance, low-alpha lattice has brought considerable benefit to users interested in time-resolved science at Diamond Light Source. The emittance for the new lattice is only marginally above that of the standard user optics at $4.4 \mathrm{~nm}$ rad compared to $2.7 \mathrm{~nm}$ rad, demonstrating both short pulses and low-emittance beams can be produced simultaneously. Careful control of the higher-order terms in alpha allows such lattices to be implemented with good lifetime and moderate injection efficiencies.

Consistent with other facilities, we have identified several limiting factors to the minimum bunch length that can be achieved for stable user operation. These include limitations to the region of stable motion brought on by higher-order terms in alpha, increased transverse beam motion due to ground motion, bunch lengthening with current due to collective effects, and cavity gradient limitations. For Diamond, these practical effects limit the bunch length to lie in the range $1-5 \mathrm{ps}$.

Studies of coherent emission in the low-alpha operating mode are at an early stage, but it is anticipated this mode of operation will bring substantial benefit to the THz/IR user beam lines presently being developed.

\section{ACKNOWLEDGMENTS}

We thank J. Rowland for optimizing the fast orbit feedback for operation in low-alpha mode, and are grateful to the Operations and RF Groups for support during machine development periods. In addition, we would like to thank G. Wüstefeld for his advice and support throughout this work.

[1] D. Robin et al., Phys. Rev. E 48, 2149 (1993).

[2] H. Hama, S. Takano, and G. Isoyama, Nucl. Instrum. Methods Phys. Res., Sect. A 329, 29 (1993).

[3] A. Nadji et al., Nucl. Instrum. Methods Phys. Res., Sect. A 378, 376 (1996).

[4] D. Robin, R. Alvis, A. Jackson, R. Holtzapple, and B. Podobedov, Report No. SLAC-PUB-95-7015, 1995.

[5] D. Robin, H. Hama, and A. Nadji, in Proceedings of the Micro Bunches Workshop, Upton, NY, 1995, AIP Conf. Proc. No. 367 (AIP, New York, 1995).

[6] J. Feikes, K. Holdack, P. Kuske, and G. Wüstefeld, in Proceedings of the 9th European Particle Accelerator Conference, Lucerne, 2004 (EPS-AG, Lucerne, 2004).

[7] A-S. Müller et al., in Proceedings of the 21st Particle Accelerator Conference, Knoxville, 2005 (IEEE, Piscataway, NJ, 2005).

[8] X. Huang, J. Safranek, J. Corbett, Y. Nosochkov, J. Sebek, and A. Terebilo, in Proceedings of the 22nd Particle Accelerator Conference, Albuquerque, New Mexico (IEEE, New York, 2007).

[9] Y.-R. E. Tan, R. Dowd, M. Boland, and D. Appadoo, in Proceedings of the 23rd Particle Accelerator Conference, Vancouver, Canada, 2009 (IEEE, Piscataway, NJ, 2009).

[10] L. O. Dallin and M. S. de Jong, in Proceedings of the 23rd Particle Accelerator Conference, Vancouver, Canada, 2009 (Ref. [9]).

[11] G. Brandt et al., Nucl. Instrum. Methods Phys. Res., Sect. B 258, 445 (2007).

[12] N. P. Abreu et al., in Proceedings of the 23rd Particle Accelerator Conference, Vancouver, Canada, 2009 (Ref. [9]).

[13] J. Feikes et al., Phys. Rev. ST Accel. Beams 14, 030705 (2011).

[14] M. Venurini, R. Warnock, R. Ruth, and J. Ellison, Phys. Rev. ST Accel. Beams 8, 014202 (2005).

[15] J. Byrd et al., Phys. Rev. Lett. 89, 224801 (2002). 
[16] R. Bartolini, M. Borland, and K. Harkay, in Proceedings of the 10th European Particle Accelerator Conference, Edinburgh, Scotland, 2006 (EPS-AG, Edinburgh, Scotland, 2006).

[17] R. Bartolini, in Procedings of the ICFA Mini-Workshop on Frontiers of Short Bunches in Storage Rings, Frascati, 2005, http://www.lnf.infn.it/conference/sbsr05/prog.html.

[18] I. P. S. Martin, R. Bartolini, J. Rowland, B. Singh, and C. Thomas, in Proceedings of the 23rd Particle Accelerator Conference, Vancouver, Canada, 2009 (Ref. [9]).

[19] H. Ehrke et al., Nature Mater. (to be published).

[20] Y. Shoji, Phys. Rev. ST Accel. Beams 8, 094001 (2005).

[21] H. Weidemann, Particle Accelerator Physics I (SpringerVerlag, Berlin, 2003).

[22] A. Nadji, in Proceedings of the 2nd Workshop on Nonlinear Beam Dynamics in Storage Rings, Diamond Light Source, 2009, http://www.diamond.ac.uk/Home/ Events/Past_events/NBD_workshop.html.

[23] M. Attal, Ph.D. thesis, Université Paris Sud 11, 2009.

[24] I. P. S. Martin, R. Bartolini, G. Rehm, J. H. Rowland, and C. Thomas, in Proceedings of the First International Particle Accelerator Conference, Kyoto, Japan (2010), http://accelconf.web.cern.ch/AccelConf/IPAC10/.

[25] J. Laskar, in Proceedings of the 20th Particle Accelerator Conference, Portland, OR, 2003 (IEEE, New York, 2003), pp. 378-382.

[26] J. Bengtsson (unpublished).

[27] M. Borland, Advanced Photon Source LS-287 (2000).
[28] A. Terebilo, Report No. SLAC-PUB-8732, 2001.

[29] C. Thomas and G. Rehm, in Proceedings of the 10th European Particle Accelerator Conference, Edinburgh, Scotland, 2006 (Ref. [16]).

[30] C. Thomas, G. Rehm, and I. Martin, in Proceedings of the First International Particle Accelerator Conference, Kyoto, Japan (2010), http://accelconf.web.cern.ch/ AccelConf/IPAC10/.

[31] G. Stupakov and S. Heifets, Phys. Rev. ST Accel. Beams 5, 054402 (2002).

[32] S. Fang, K. Oide, K. Yokoya, B. Chen, and J. Wang, KEK Report No. 94-190, 1995.

[33] J. Rowland, R. Bartolini, R. Fielder, and R. Nagaoka, in Proceedings of the First International Particle Accelerator Conference, Kyoto, Japan (2010), http://accelconf .web.cern.ch/AccelConf/IPAC10/.

[34] G. Wüstefeld, in Proceedings of the 11th European Particle Accelerator Conference, Genoa, 2008 (EPS-AG, Genoa, Italy, 2008).

[35] G. Rehm, A. Morgan, R. Bartolini, I. Martin, and P. Karataev, in Proceedings of DIPAC 2009 (2009), http:// dipac09.web.psi.ch/Proceedings/index.htm.

[36] J. Feikes, P. Kuske, R. Müller, and G. Wüstefeld, in Proceedings of the 10th European Particle Accelerator Conference, Edinburgh, Scotland, 2006 (Ref. [16]).

[37] C. Thomas, R. Bartolini, I. Martin, and G. Rehm, Phys. Rev. ST Accel. Beams 13, 022805 (2010). 